Debate

Series editors: Susan Lightman and Peter McCluskey

Correspondence to: M Daniell, Royal Victorian Eye and Ear Hospital, Melbourne, Victoria, 3065, Australia;

daniellm@ozemail.com.au

Accepted for publication 1 May 2003

CONTROVERSIES IN OPHTHALMOLOGY

\title{
Microbial keratitis: what's the preferred initial therapy?
}

\author{
M Daniell, R Mills, N Morlet
}

A 29 year old woman, who was an intermittent contact lens wearer, is referred from her local ophthalmologist with a diagnosis of microbial keratitis. There is a large central corneal ulcer with stromal white cell recruitment and low grade anterior chamber (AC) activity. The patient has no other eye problems and is in good health.

Would you perform a corneal scraping? Which antibiotics would you use to begin empirical treatment for her microbial keratitis?

\section{View 1: Corneal scraping and combination antibiotic therapy is indicated}

\section{R Mills}

M icrobial keratitis is an ocular emergency that requires prompt and appropriate management to ensure the best visual outcome for the patient. If possible, this means identification of the causative organism(s) and selection of the best antimicrobial agent(s) to treat the patient.

A clinical diagnosis of corneal infection does not give an unequivocal indication of the causative organism because a wide range of organisms can produce a similar clinical picture. The clinical history together with the duration and severity of symptoms and signs may make the clinical diagnosis of one type of organism more likely, but without attempting to identify the causative organism(s) treatment can only be based on clinical judgment.

The identification of organisms responsible for microbial keratitis is desirable because a positive result indicates the appropriate class of antimicrobial agent to use. Knowing the causative organism also gives the treating clinician confidence to pursue a particular line of therapy and avoids the unnecessary use of ineffective and potentially toxic drugs. Culturing allows sensitivity testing to a range of agents so that treatment modification can be made in an informed manner if the clinical response to initial treatment is inadequate.

\section{BACTERIAL KERATITIS}

In urban communities of developed countries the most common bacteria causing keratitis are Staphylococcus, Streptococcus, and Pseudomonas species. For more than 20 years the recommended initial therapy for bacterial keratitis has centred around the use of topical broad spectrum fortified antibiotics that cover these bacteria. ${ }^{1}$ The usual choice is between a first generation cephalosporin (for example, cefazolin 5\%) to cover the Gram positive cocci and an aminoglycoside (for example, gentamicin $1 \%$ ) to cover the Gram negative organisms. These antibiotics could be used either alone or in combination, depending on the results of laboratory identification. ${ }^{1}$ This therapy was based on data from the treatment of experimental Pseudomonas keratitis which showed that increasing both the frequency of application and the concentration of topical antibiotics increased the antibiotic concentrations in the cornea and improved the therapeutic response. ${ }^{2-4}$ Clinical data spanning a 20 year period confirm that this approach has been highly successful. ${ }^{56}$ Over the past decade the most significant variation to this approach has occurred following the introduction of topical fluoroquinolone antibiotics.

Competing manufacturers of topical fluoroquinolone antibiotics were quick to support clinical trials of bacterial keratitis treatment. ${ }^{7-11}$ These well designed studies were done to compare the efficacy and safety of commercially available fluoroquinolone antibiotics with combination fortified cephalosporin and aminoglycoside antibiotics. The conclusions from these studies, together with other retrospective comparisons, ${ }^{6}$ show no difference in the treatment success between the two therapies. What these studies do highlight is the difficulty in defining a cure for infectious keratitis. The aim of antibiotic treatment is sterilisation of the cornea, but this does not necessarily equate with healing of the epithelium or resolution of inflammation. ${ }^{12}$ Closure of the epithelium is, however, used as a significant end point in defining a cure in these comparative studies, ${ }^{7-11}$ and may lead to an overestimation of treatment failure. The studies also demonstrate that "failures" 


\section{Debate}

occur with all therapies and are not necessarily associated with organism susceptibility to the antibiotic given. Clearly, many factors such as disease severity, drug toxicity, stromal inflammation, and dysfunction of the external eye may influence the resolution of an episode of infectious keratitis. Nevertheless, topical fluoroquinolone antibiotics have become popular in the treatment of bacterial keratitis based on the results of these studies.

Fluoroquinolone antibiotic drops have been recommended for bacterial keratitis ${ }^{7-11}$ because of their ease of availability, broad spectrum of activity, and apparent lack of toxicity. There are some concerns however. Clinical and laboratory reports show emerging resistance and gaps in the spectrum of activity for organisms that commonly cause keratitis. ${ }^{13}{ }^{14}$ Experimental Streptococcus pneumoniae keratitis has shown that topical penicillin and gentamicin are best for treating keratitis caused by penicillin sensitive and penicillin resistant $S$ pneumoniae, respectively. ${ }^{15} \mathrm{Re}-$ views of antibiotic susceptibilities of bacteria isolated from cases of keratitis have even prompted the recommendation of combining fluoroquinolones with an antibiotic with superior Gram positive activity such as cefazolin. ${ }^{16}$ The problem with reviewing in vitro susceptibility testing is that is does not relate to treatment success or failure.$^{17}$ One of the most likely reasons for this discrepancy is the high concentration of antibiotics achievable by topical delivery in the inflamed ulcerated cornea.

A more serious complication reported with the use of topical ofloxacin is an increased incidence of corneal perforation. ${ }^{6}{ }^{18}$ This has not been verified in clinical trials but remains a significant concern. The possible mechanism is not understood but experimentally ofloxacin, ciprofloxacin, and norfloxacin have been shown to have significantly higher antiproliferative and cytotoxic effects than gentamicin and tobramycin on rabbit keratocytes in vitro. ${ }^{19}$

Another potential concern is that the commercial availability of fluoroquinolones has tended to encourage empirical treatment of infectious keratitis and discourage investigation. ${ }^{20}$ Such an approach is problematic for individual cases that are not caused by bacteria or are non-responsive to initial treatment because it delays diagnosis and exposes the patient to inappropriately prolonged and potentially toxic treatment. This approach also will ultimately limit knowledge related to the epidemiology of organisms causing keratitis and, subsequently, make recommendations on primary therapy difficult.

Both fortified antibiotics and fluoroquinolones are effective for the treatment of bacterial keratitis. Each mode of therapy provides good cover for the most frequently encountered bacteria causing keratitis with combination fortified antibiotics providing a theoretically superior Gram positive cover. The commercial availability and longer shelf life of fluoroquinolone antibiotics make them more convenient to use. Greater short term epithelial toxicity occurs with fortified antibiotics, particularly the aminoglycosides, but the more serious potential for increased rate of corneal perforation with ofloxacin needs to be monitored. I think the best approach to the initial treatment of bacterial keratitis remains combination fortified antibiotics with the use of fluoroquinolones, an approach that is as effective but not superior to fortified antibiotics.

\section{FUNGAL AND ACANTHAMOEBA KERATITIS}

There is far less controversy related to the selection of antimicrobial agents for the treatment of fungal and acanthamoeba keratitis. However, because of antimicrobial toxicity and the possible need for prolonged therapy, treatment should not be started until microscopy or culture of samples taken from the cornea identifies organisms.

Clinical and experimental studies have shown that topical natamycin 5\% is the drug of choice for filamentous fungal keratitis. ${ }^{21}$ Topical amphotericin B $0.15 \%$ is the preferred initial agent for yeast infection. The triazole fluconazole is also effective topically as a $0.2 \%$ solution for candida keratitis. ${ }^{22}$ Fluconazole and itraconazole can also be administered orally. ${ }^{23}{ }^{24}$

Acanthamoeba keratitis should initially be treated with a combination of propamidine isethionate $0.1 \%$ (Brolene) and polyhexamethylene biguanide $0.02 \%$ (PHMB). ${ }^{25}$ Brolene is easily obtainable in the United Kingdom and Australia, whereas PHMB needs to be made up on an individual basis and can be difficult to obtain.

\section{REFERENCES}

1 Jones DB. Decision making in the management of microbial keratitis. Ophthalmology 1981;88:814-20.

2 Davis SD, Sarff LD, Hyndiuk RA. Antibiotic therapy of experimental Pseudomonas keratitis in guinea pigs. Arch Ophthalmol 1977;95:1638-43.

3 Davis SD, Sarff LD, Hyndiuk RA. Topical tobramycin therapy of experimental Pseudomonas keratitis. An evaluation of some factors that potentially enhance efficacy. Arch Ophthalmol 1978;96:123-5.

4 Kuperfman A, Leibowitz HM. Topical antibiotic therapy of Pseudomonas aeruginosa keratitis. Arch Ophthalmol 1979;97:1699-702

5 Jones DB. Strategy for the initial management of suspected microbial keratitis. In: symposium on medical and surgical diseases of the cornea. Transactions of the New Orleans Academy of Ophthalmology. St Lovis: CV Mosby, 1980:86-119.

6 Gangopadhyay N, Daniell M, Weih L, et al. Fluoroquinolone and fortified antibiotics for treating bacterial corneal ulcers. $\mathrm{Br} J$ Ophthalmol 2000;84:378-84.

7 Leibowitz H. Clinical evaluation of ciprofloxacin $0.3 \%$ ophthalmic solution for treatment of bacterial keratitis. Am J Ophthalmol 1991:112:34S-47S.

8 Wilhelmus KR, Hyndiuk R, Caldwell D, et al. $0.3 \%$ Ciprofloxacin ophthalmic ointment in the treatment of bacterial keratitis. Arch Ophthalmol 1993;111:121018

9 O'Brien TP, Maguire MG, Fink NE, et al. Efficacy of ofloxacin vs cefazolin and tobramycin in the therapy for bacterial keratitis. Arch Ophthalmol 1995;113:125765.

10 Hyndiuk RA, Eiferman RA, Caldwell DR et al. Comparison of ciprofloxacin ophthalmic solution $0.3 \%$ to fortified tobramycin-cefazolin in treating bacterial corneal ulcers. Ophthalmology 1996;103: 1854-63.

11 Ofloxacin Study Group. Ofloxacin monotherapy for the primary treatment of microbial keratitis. A double-masked, randomized, controlled trial with conventional dual therapy. Ophthalmology 1997; 104:1902-9.

12 Allan BD, Dart JK. Strategies for the management of microbial keratitis. Br J Ophthalmol 1995;79:777-86.

13 Goldstein M, Kowalski M, Gordon G. Emerging fluoroquinolone resistance in bacterial keratitis. Ophthalmology 1999;106:1313-18.

14 Garg P, Sharma S, Rao G. Ciprofloxacin-resistant Pseudomonas keratitis. Ophthalmology 1999:106:1319-23. 
15 Guzek JP, Cline DJ, Row PK, et al. Rabbit Streptococcus pneumoniae keratitis model and topical therapy. Invest Ophthalmol Vis Sci 1998;39:2012-17

16 Bower KS, Kowalski RP, Gordon YJ. Fluoroquinolones in the treatment of bacterial keratitis. Am J Ophthalmol 1996:121:712-15.

17 Jones DB. Emerging antibiotic resistance: real and relative. Arch Ophthalmol 1996;1 14:91-2.

18 Mallari P, McCarty D, Daniell $M$, et al. Increased incidence of corneal perforation after topical fluoroquinolone treatment for microbial keratitis. Am J Ophthalmol 2001;131:131-3

19 Seitz B, Hayashi S, Wee W, et al. In vitro effects of aminoglycosides and fluoroquinolones on keratocytes. Invest Ophthalmol Vis Sci 1996;37:656-65.
20 McLeod S, DeBacker C, Viana M. Differential care of corneal ulcers in the community based on apparent

21 O'Day DM. Fungal keratitis. In: Fraunfelder FT, Roy FH, eds. Current ocular therapy. Philadelphia: WB'Saunders, 2000:360-1

22 Panda A, Sharma N, Angra S. Topical fluconazole therapy of Candida keratitis. Cornea 1996;15:373-5

23 Thakar $M$. Oral fluconazole therapy for keratomycosis. Acta Ophthalmol 1994;72:765-7.

24 Koltz S, Zahid M, Bartholomew W, et al. Candida albicans keratitis treated successfully with itraconazole. Cornea 1996:15:102-4.

25 Duguid IG, Dart JK, Morlet N, et al. Outcome of Acanthamoeba keratitis treated with polyhexamethyl biguanide and propamidine. Ophthalmology 1997:104:1587-92.

\title{
View 2: Empirical fluoroquinolone therapy is sufficient initial treatment
}

\author{
N Morlet, M Daniell
}

A lthough the treatment of microbial keratitis has changed with the introduction of new antimicrobials, the management principles still remain the same. In general, suspected microbial keratitis is treated with empirical therapy of intensive topical broad spectrum antimicrobials. This is because delaying treatment until the diagnosis is confirmed may worsen the visual outcome and allow further complications. Whether there is a need for microbiological investigation for all patients is contentious, as is empirical primary treatment with fluoroquinolone monotherapy.

\section{WHAT CAUSES MICROBIAL KERATITIS?}

Microbial keratitis is rare in the absence of predisposing risk factors. In the past trauma and ocular surface compromise (for example, bullous keratopathy, exposure, etc) were the major risks. However, with the introduction of soft contact lenses and their widespread use since the 1980s, the demographic profile of those presenting with suspected microbial keratitis has changed. Figure l shows the demographic change in the age groups of those presenting with suspected microbial keratitis from 1985 to 1995 at Moorfields Eye Hospital in London, United Kingdom.

The change in the presentation of suspected microbial keratitis over time was also reflected in the types of micro-organisms cultured as shown in Table 1.

Not only are there temporal changes in the pattern of presentation of microbial keratitis, there are also geographic differences in the pattern of presentation. Table 2 shows the different patterns of infection from reports of keratitis from various locations around the world.

So it is evident that the empirical choice of antibiotics in the primary treatment of suspected microbial keratitis requires local contemporaneous data regarding the spectrum of disease. For such data to be collected it is necessary for patients to have a microbiological investigation, which not only provides information on the pattern of presentation, but may also provide important information regarding the change in antibiotic sensitivities with time.

\section{DOES THIS MEAN THAT EVERY PATIENT SHOULD BE SCRAPED FOR \\ MICROBIOLOGICAL INVESTIGATION?}

Certainly there is evidence that this not the norm among general ophthalmologists in the United States. A survey by McDonnell in 1992 found that only $14(23 \%)$ of 64 randomly selected general ophthalmologists considered a scrape necessary all the time. ${ }^{1}$ Perhaps the change in presentation of suspected microbial keratitis at Moorfields provides part of the explanation; there are more young patients, probably contact lens wearers, often with a negative corneal scrape culture. The availability of potent monotherapy "off the shelf"
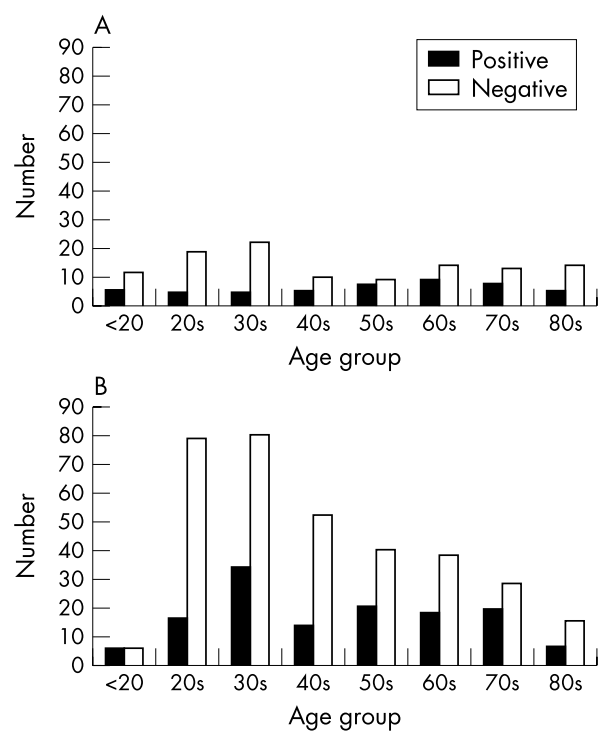

Figure 1 (A) Corneal scrape results by age group, Moorfields Eye Hospital, 1985. (B) Corneal scrape results by age group, Moorfields Eye Hospital, 1995.

Debate

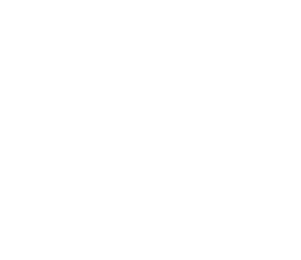

\section{.}


Table 1 Variation in the organisms cultured from corneal scrapes at Moorfields Eye Hospital from 1985 to 1995

\begin{tabular}{lllllrr}
\hline & Staphylococcus sp & Streptococcus sp & Pseudomonas & Gram negatives & Acanthomoeba & Fungi \\
\hline 1985 & $29 \%$ & $22 \%$ & $19 \%$ & $25 \%$ & $0 \%$ & $13 \%$ \\
1995 & $34 \%$ & $13 \%$ & $21 \%$ & $12 \%$ & $13 \%$ & $6 \%$ \\
\hline
\end{tabular}

Table 2 Regional variation in the organisms cultured from corneal scrapes

\begin{tabular}{lcccrr}
\hline & Staphylococcus & Streptococcus & Pseudomonas & \multicolumn{2}{c}{ Gram negatives } \\
& & $9 \%$ & $8 \%$ & $22 \%$ & $3 \%$ \\
\hline New York, USA & $49 \%$ & $8 \%$ & $19 \%$ & $9 \%$ & $35 \%$ \\
Florida, USA & $16 \%$ & $29 \%$ & $4 \%$ & $14 \%$ & $3 \%$ \\
South Africa & $45 \%$ & $37 \%$ & $11 \%$ & $14 \%$ & $17 \%$ \\
Nepal & $23 \%$ & $24 \%$ & $22 \%$ & $5 \%$ & $45 \%$ \\
Bangladesh & $2 \%$ & $13 \%$ & $7 \%$ & $14 \%$ & \\
Melbourne, Australia & $48 \%$ & & &
\end{tabular}

has also allowed general ophthalmologists to treat suspected microbial keratitis successfully without the need to refer patients to corneal specialists who would be more likely to use microbiological investigation and extemporaneous fortified topical antibiotic preparations.

The yield from microbiological investigation may be low, despite the direct inoculation of the sample onto culture media at the time of the scrape. Reviewing the results from 18 published studies (3836 patients), a positive culture averaged 51\% (95\% confidence interval 34-67), and a positive Gram stain averaged 67\% (95\% CI 60 to $75)$. There are probably a number of reasons for the low yield, such as the variability in the diagnosis, operator skill producing a variable quality sample, and techniques of culture, to name but a few. However, the ulcer size definitely has an effect on the culture yield. In the UK Ofloxacin Study ${ }^{2}$ ulcer size was a significant influencing factor for the 49 of 118 (42\%) patients that were culture positive (Table 3), suggesting that the microbiological investigation of corneal ulcers less than $2.0 \mathrm{~mm}^{2}$ in size is probably not worthwhile.

The risk of primary treatment failure varied in previous reports from $4 \%$ to $28 \%$, the UK Ofloxacin Study found that 12\% (14 of 118) failed primary treatment, and 10 of the 14 required some form of surgical intervention. Those with persistent or indolent ulceration (15 of 188, 13\%) were found to have previous ocular surface disease (relative risk $12.65,95 \%$ CI 1.72 to 93.1 ), or previous topical steroid use (RR 4.61, 95\% CI 1.88 to 11.08 ). Slowly healing ulcers (17 patients, $14 \%$ ) were also related to ocular surface disease (RR $14.5,95 \%$ CI 1.98 to 105.48 ) but also a positive culture (RR $4.58,95 \%$ CI 1.59 to 13.2 ). ${ }^{2}$
Thus, the results from the UK Ofloxacin Study suggest that young patients with small ulcers do well and are unlikely to be culture positive. However, large $\left(>5 \mathrm{~mm}^{2}\right)$ culture positive ulcers in elderly patients ( $>60$ years old) had 5.5 times the risk of primary treatment failure than others. Culture positive ulcers were also more likely to take longer to heal. ${ }^{2}$

For the individual patient, a positive culture result from microbiological investigation is more often useful as a prognostic indicator than having diagnostic significance, given that $80 \%+$ will be treated satisfactorily with empirical broad spectrum antibiotics. However, as already stated, the combined local results from microbiological investigation will guide the ophthalmologist's choice of empirical therapy in the first place. When an individual fails primary treatment, an initial positive culture result will guide the choice of secondary therapy, perhaps reducing further delay in resolution of the infection.

\section{SHOULD PRIMARY TREATMENT OF SUSPECTED MICROBIAL KERATITIS RELY ON FLUOROQUINOLONE MONOTHERAPY?}

Obviously the local demographic and the clinical presentation must influence the choice of therapy. In urban societies, for those unlikely to fail primary treatment (that is, young patients with a small ulcer), monotherapy with an "off the shelf" preparation is a convenient low risk option. This would seem to be common practice as the McDonnell survey suggested.

For the "at-risk" population, the elderly with large ulcers and previous ocular surface disease, ciprofloxacin and ofloxacin monotherapy were as

Table 3 Distribution of ulcer sizes and their culture results from the UK Ofloxacin Study ${ }^{2}$

\begin{tabular}{lllr}
\hline & 25th quartile & Median & 75th quartile \\
\hline Positive culture & $2.00 \mathrm{~mm}^{2}$ & $6.0 \mathrm{~mm}^{2}$ & $12.00 \mathrm{~mm}^{2}$ \\
Negative culture & $0.25 \mathrm{~mm}^{2}$ & $1.0 \mathrm{~mm}^{2}$ & $2.25 \mathrm{~mm}^{2}$ \\
\hline p<0.0001 Kruskal-Wallis H. & & & \\
\hline
\end{tabular}


effective as the previous convention (fortified gentamicin combined with a fortified cephalosporin) in a number of randomised controlled trials. ${ }^{3-5}$ However, if there are concerns about the possibility of a pneumococcal infection or there are resistant Gram positive organisms within the local demographic, a combination of the fluoroquinolone and a fortified cephalosporin would be a better alternative to fortified gentamicin. Although efficacious, fortified aminoglycosides are considerably more toxic to the ocular surface than fluoroquinolones, and may result in delayed healing, and other problems. Cephazolin 5\% would be a good choice-add $2.5 \mathrm{ml}$ to $1000 \mathrm{mg}$ cephazolin powder then add to $17 \mathrm{ml}$ Liquifilm Tears; this will be stable for 28 days if refrigerated.

In the UK Ofloxacin Study resistant organisms were found in both treatment arms of the trial, and primary treatment failure occurred equally as often for the conventional therapy (fortified cefuroxime and gentamicin) as it did for ofloxacin monotherapy. ${ }^{2}$ So it is important to be able to recognise those patients at risk of primary treatment failure (for example, the elderly with large ulcers), and have a management algorithm that identifies and manages those treatment failures early, regardless of the initial treatment instituted.

\section{OTHER MANAGEMENT ISSUES}

Often it is much easier to start than to stop the treatment. The signs of resolution are subtle; however, the symptoms are often a good early guide. Toxicity from the treatment may obscure signs of resolution, particularly if the initial intensive treatment is overly prolonged and utilises toxic antibiotics such as the aminoglycosides. So treatment should be considered as two phases-the initial intensive treatment to sterilise the cornea, followed by the healing phase, with prophylactic doses of antimicrobials to prevent further infection.

Initial treatment should be intensive with hourly application of antibiotic to "marinade" the eye so that the corneal tissue is rapidly saturated with a high antibiotic concentration. A high concentration (usually exceeding the minimum inhibitory concentration (MIC) by a number of $\log$ units) can be achieved within a few hours, so that 48 hours of sustained high concentrations is usually enough to eliminate most bacterial infections, sometimes even those organisms only partly sensitive to the antibiotic. Sustained intensive treatment day and night for the first 48 hours, then hourly by day for the next 2-3 days, would allow for more than an adequate chance of sterilising the corneal ulcer. Admission to hospital to ensure compliance and observe the clinical response is frequently required for the elderly with large ulcers, particularly if there is corneal thinning. For smaller shallow ulcers a shorter intensive period may be adequate, and these may be managed on an outpatient basis. The results from any corneal scrape, with sensitivities, may be used to rationalise or modify treatment at 48 hours. It is worthwhile adding oral doxycycline to the therapy if the ulcer is large and there is corneal thinning. At a dose of $100 \mathrm{mg}$ twice a day, doxycycline is a metalloproteinase inhibitor and may help prevent corneal perforation. ${ }^{6}$
Lack of clinical response requires secondary management which is best undertaken by corneal specialists, especially if an exotic organism is involved or if corneal perforation is impending.

Following the initial treatment phase the antibiotic application is reduced to four times a day to allow for healing of the epithelial defect. Tapering the initial therapy offers no clinical advantage and is only likely to increase the likelihood of toxicity. The healing phase may be prolonged for large culture positive ulcers, especially in the elderly who may also have ocular surface disease. It is important to optimise the ocular surface environment, correcting any factors such as aqueous tear deficiency, Meibomian gland disease, exposure, etc. Topical steroids are often required to settle the resultant inflammation from the keratitis and may ultimately be necessary to promote healing of the epithelial defect. ${ }^{78}$ These are used with caution in those patients who had fungal keratitis; however, a pronounced inflammatory response from a fungal infection may be difficult resolve otherwise. Poor healing ulcers may require management by a corneal specialist.

\section{CONCLUSIONS}

The initial management of suspected microbial keratitis with intensive empirical antimicrobials is largely successful with whatever primary management regime used. However, the unique combination of pathogen and host response may result in an adverse outcome despite optimal management. ${ }^{9}$ Vigilance and a secondary management algorithm for such cases is required. It is the early identification of those at risk, and those that are failing the initial management, that will prevent loss of the eye and ultimately improve the chance of an acceptable visual outcome for the patient.

\section{REFERENCES}

1 McDonnell PJ, Nobe J, Gauderman WJ, et al. Community care of corneal ulcers. Am J Ophthalmol 1992;114:531-8.

2 Morlet N, Minassian D, Butcher J. Risk factors for treatment outcome of suspected microbial keratitis. Ofloxacin Study Group. Br J Ophthalmol 1999;83:1027-31

3 Parks DJ Abrams DA, Sarfarazi FA, et al. Comparison of ciprofloxacin to conventional antibiotic therapy in the treatment of ulcerative keratitis. Am J Ophthalmol 1993;1 15:471-7

4 O'Brien TP, Maguire MG, Fink NE, et al and the Bacterial Keratitis Study Research Group. Efficacy of ofloxacin vs cefazolin and tobramycin in the therapy for bacterial keratitis. Arch Ophthalmol 1995;113:125765.

5 Ofloxacin Study Group. Ofloxacin monotherapy for the primary treatment of suspected microbial keratitis. Ophthalmology 1997;104:1902-9.

6 Ralph RA. Tetracyclines and the treatment of corneal stromal ulceration. Cornea 2000;19:274-7.

7 Leibowitz Hm, Kupferman A. Topically administered corticosteroids. Effect on antibiotic-treated bacterial keratitis. Ophthalmology 1980;98:1287-90.

8 Stern GA, Buttross M. Use of corticosteroids in combination with antimicrobial drugs in the treatment of infectious corneal disease. Ophthalmology $1991 ; 98: 847-53$

9 Coster DJ, Badenoch PR. Host, microbial and pharmacological factors affecting the outcome of suppurative keratitis. Br J Ophthalmol 1987;71:96-101.
Debate 


\section{Debate}

FURTHER READING

The American Academy of Ophthalmology. Preferred practice pattern bacterial keratitis. San Francisco: AAO.

Allen BDS, Dart JKG. Strategies for the management of microbial keratitis. Br J Ophthalmol 1995;79:777-86.
Neu HC. Microbiological aspects of fluoroquinalones. Am J Ophthalmol 1991;112:15S-24S.

Wong TTL, Sethi C, Daniels JT, et al. Mattrix metalloprotienases in disease and repair processes in the anterior segment. Surv Ophthalmol 2002;47:239-56

\section{Overview: Initial antimicrobial therapy for microbial keratitis}

\section{Daniell}

E mpirical antibacterial therapy in keratitis is based on the likely pathogen, the available drugs, and the severity of the condition. ${ }^{1}$ Ongoing treatment is modified by clinical response and the result of initial microbial investigations. ${ }^{2}$

There is a large degree of variation in organism type and their resistance patterns from centre to centre, and so local contemporaneous data are essential to make a rational choice of initial antibiotic therapy. Reference centres specialising in corneal disease with good microbiological backup have a key role in analysing local trends and disseminating their results. Ongoing audit is also required, as patterns of resistance are invariably changing.

Overall, there are some common pathogens that cause acute bacterial keratitis. Streptococcal species, staphylococcal species, pseudomonas and enterobacteriaceae make up the four most common classes of infective agents and any empirical therapy has to cover all these groups.

\section{STREPTOCOCCAL SPECIES}

There have been some concerns about the lack of in vitro efficacy by fluoroquinolone in the streptococcal species. ${ }^{45}$ In practical terms, this has not proved to be a common problem to date in the United Kingdom and Australia. ${ }^{6}$ In vitro resistance is based on the $\mathrm{MIC}_{90}$ and assumes drug levels found following intravenous infusion. However, topical administration can produce far

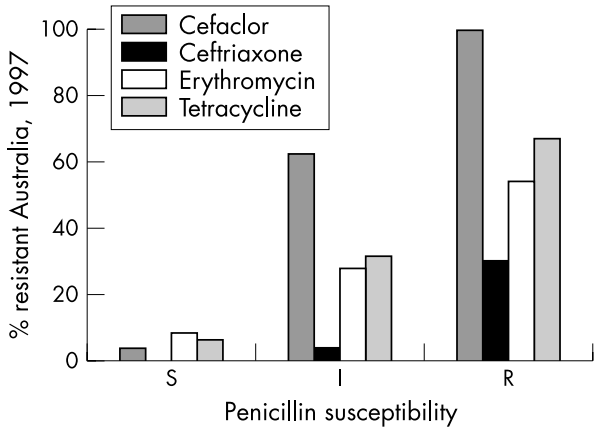

Figure 1 Penicillin and other drug resistance in Streptococcus pneumoniae. Resistance rates for sensitive (S), intermediate (I), and resistant (R) strains to various antibiotics. Overall, rate of penicillin resistance in 1989 was $1 \%$. In 1997 the rate of penicillin resistance was $25 \%$. higher tissue levels in the cornea than those obtained by intravenous administration and can be effective in local control of streptococcal keratitis. That being said, there will be occasions where the $\mathrm{MIC}_{90}$ will be at such a level that topical administration cannot control the disease and we have certainly had clinical experience of resistant streptococci. Newer methods to assess in vitro sensitivity other than the Kirby-Bauer test that can provide a more clinically relevant definition of resistance need to be introduced.

Penicillin or cephalosporins have been traditionally recommended for streptococcal infection and are effective alternatives in keratitis.

There is, however, emergence of penicillin resistant pneumococci. Studies in Australia reveal levels of $25-30 \%$ of penicillin resistance and 34\% of US isolates are resistant (Fig l). ${ }^{7}$

Based on these data and the prospect of emerging clinical resistance in keratitis patients, cephalosporins and penicillin will have a limited role in the future. Ciprofloxacin is not highly effective against streptococcal species. Newer quinolones such as gatifloxacin and moxifloxacin are much more rational choices.

\section{STAPHYLOCOCCAL SPECIES}

Staphylococcal species have been traditionally susceptible to $\beta$ lactam drugs such as cephalosporin. Non-resistant strains show a high susceptibility to quinolones. Most series of bacterial keratitis show good efficacy with either of these choices. Resistant cases need vancomycin, which can be applied topically. Multiple resistant Staphylococcus aureus has become an increasing problem, particularly in nosocomial infection (Fig $2){ }^{8}$

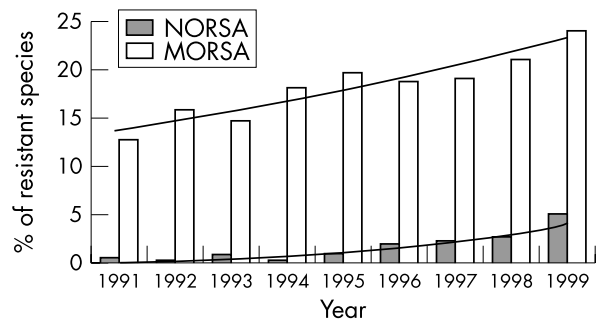

Figure 2 Change in rate of methicillin resistant Staphylococcus aureus. Australian Group on Antimicrobial Resistance (AGAR). NORSA = not multiresistant Staphylococcus aureus; MORSA = multiresistant Staphylococcus aureus. 


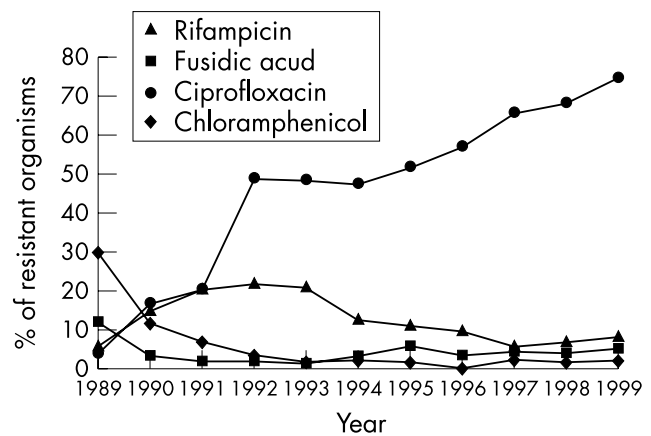

Figure 3 Emerging resistance in MRSA (methicillin resistant Staphylococcus aureus) 1989-99 (AGAR). Incidence of resistant strains to various antibiotics plotted annually.

These MRSA (methicillin resistant Staphylococcus aureus) species have developed new patterns of resistance to antibiotics, particularly to the fluoroquinolones (Fig 3).

These figures are similar if not worse in the United States.

Ciprofloxacin is suitable for most staphylococcal species but not MRSA. If this becomes a more common cause of keratitis, then use of newer agents such as gatifloxacin and moxifloxacin will need to be considered. ${ }^{9}$

\section{PSEUDOMONAS SPECIES}

Pseudomonas is one of the most potent pathogens of the cornea and is relatively common in some populations particularly the United Kingdom. Aminoglycosides have been highly effective and resistance is uncommon. ${ }^{10}$ However, the prolonged treatment required and the tissue damage associated with the infection make the toxic side effects of treatment a common and difficult management issue. ${ }^{211}$

Quinolones have proved highly effective against pseudomonas to date. ${ }^{12} 13$

True resistance to ciprofloxacin is emerging particularly in India. ${ }^{14}$

The newer agents gatifloxacin and moxifloxacin do not have the same potency against pseudomonas, which may prove a problem if they are to be used as monotherapy. ${ }^{10}$

\section{ENTEROBACTERIACEAE}

This is the other common group of organisms that cause microbial keratitis. Cephalosporins are less effective than aminoglycosides which have been the mainstay of treatment. Quinolones are highly effective (Table 1).

Debate

\section{SUMMARY}

Fluoroquinolones are used as monotherapy in many centres and are highly effective (Table 1).

Tissue concentration can vary dramatically depending on associated conditions and so can make estimation of the true tissue concentration difficult. The determinants of corneal and aqueous antibiotic concentration are based on a standard concentration of drug and frequent intensive applications. However other factors such as the presence of epithelial defect and the formulation can markedly alter penetration of drug into the cornea. As previously alluded to, despite the theoretical relative resistance of streptococcal species to quinolones, clinical practice has not revealed a poor response to topical quinolones in most cases of streptococcal keratitis. Only in a small group of patients does in vitro resistance translate to poor efficacy in vivo. Both prospective trials such as the Ofloxacin Study and retrospective audit have shown equivalent overall efficacy of combined fortified and quinolone therapies. ${ }^{13} 1617$

This lack of in vivo resistance has been explained as being a consequence of higher than predicted local tissue concentrations overwhelming a relative but not absolute resistance to ofloxacin. This has been confirmed in a series of more than 42 patients with culture proved streptococcal keratitis treated with ofloxacin (Proceedings of the International Conference on Ocular Infections, Jerusalem, 1995). However, as ofloxacin resistant organisms become more prevalent in the community, this situation is likely to change. A 5 year review published by Goldstein et al showed a significant increased resistance of Staph aureus to fluoroquinolones over the study period and questioned the use of monotherapy. Despite the increasing in vitro resistance in staphylococcal and streptococcal ulcers, topical therapy still produced corneal concentrations far in excess of the MIC values and therapy was only changed in $10 \%$ of cases despite up to $41 \%$ resistance rates for these isolates.

The methods used to assess in vitro sensitivity, usually the Kirby-Bauer test for bacteria, need to be modified to take into account typical tissue concentrations obtained by topical treatment, and

Table 1 Summary of estimated in vivo effective concentration of quinolones required with topical administration. Estimates of tissue concentrations obtained with oral and topical use in ideal conditions

\begin{tabular}{lll}
\hline & MIC $_{90}(\mathrm{mg} / \mathrm{ll})$ & \\
\cline { 2 - 3 } & Ciprofloxacin & Gatifloxacin/moxifloxacin \\
\hline S pneumoniae & 2 & $0.12-0.5$ \\
S aureus (all) & $0.25-2$ & $0.06-0.13$ \\
MRSA & $\mathrm{R}$ & $6.25 / 2.0$ \\
Ps aeruginosa & $0.5(8)$ & $3-32$ \\
Enterobacteriaceae & $0.01-0.25$ & $0.01-0.39$ \\
$\mathrm{C}_{\text {max }}$ (serum, $\left.\mathrm{mg} / \mathrm{ll}\right)$ & $2.4_{500 \mathrm{mg} \text { po }}$ & $4.2-4.5_{400 / 40 \mathrm{mg} \text { po }}$ \\
Aqueous $(\mathrm{mg} / \mathrm{l})$ & $0.69_{750 \mathrm{mg} \times 3 \text { po }}$ & \\
Cornea $(\mathrm{mg} / \mathrm{l})$ & $0.6_{0.3 \%}$ topical & \\
\hline
\end{tabular}


Debate

so provide more clinically relevant data. ${ }^{3}$ As resistance develops organisms will appear that will not respond even to very high local concentrations currently applied, and treatment failures will increase.

The other findings to become apparent from the clinical studies that have a significant input into clinical care are the toxicity of the medications.

Aminoglycosides produce well recognised epithelial toxicity. Prolonged intensive treatment leads to marked epithelial toxicity with pain, redness, punctate staining, and eventual retardation of epithelial healing. Non-healing epithelial defects are far more common in aminoglycoside treated keratitis. Retrospective series reveal an incidence of $5.9 \%$ of non-healing epithelial defects at 14 days in gentamicin treated eyes versus $0 \%$ in fluoroquinolone treated eyes. ${ }^{13}$

Ciprofloxacin produces white epithelial deposition in up to $16 \%$ of cases. This can mask the underlying signs and will retard epithelial healing until the drug deposits wash out. ${ }^{18}$

Retrospective audit found an increased incidence of corneal perforation in cases treated with ofloxacin. ${ }^{19}$ Fluoroquinolones are well recognised to cause spontaneous tendon rupture in otherwise well adults taking oral doses. A proposed mechanism of corneal perforation has been an increase in keratocyte apoptosis. ${ }^{20}$ Until these findings are refuted, great care should be taken in treating large deep ulcers in the elderly with ofloxacin alone.

\section{RECOMMENDATIONS FOR EMPIRICAL ANTIBACTERIAL THERAPY IN KERATITIS}

Small peripheral lesions, if not vision threatening, can be treated with quinolones alone. Corneal scrapes for culture should be taken if the patient presents to a reference centre for corneal disease.

For a large central sight threatening keratitis, corneal scrape is mandatory to both isolate resistant organisms as soon as possible and also to identify organisms that may respond poorly to initial therapy. Also this would identify unusual causes of infection earlier and allow for treatment modification.

Treatment is with quinolone plus cephalosporin, particularly in areas where streptococci and resistant staphylococci are common. Newer fluoroquinolones such as gatifloxacin and moxifloxacin offer some theoretical advantages but trials have not been completed.

Alternatively, cephazolin plus tobramycin can be used and is a reasonable choice. Increased toxicity, delayed healing, and the lack of a commercially available preparations are major deterrents.
Careful clinical and microbiological reassessment at 48 hours is essential for optimal management. A willingness to change or add antibiotics if sensitivities reveal resistance and skilful secondary management of the healing phases will determine outcome.

\section{REFERENCES}

1 Jones DB. Decision making in the management of microbial keratitis. Ophthalmology 1981;88:814-20.

2 Allen BDS, Dart JKG. Strategies for the management of microbial keratitis. Br J Ophthalmol 1995;79:777-86.

3 Tuft SJ Suppurative keratitis. Br J Ophthalmol 2003;87:127.

4 Kunimoto DY, Sharmer S, Garg P, et al. In vitro susceptibility of bacterial keratitis pathogens to ciprofloxacin. Ophthalmology 1999;106:80-5.

5 Goldstein MH, Kowolski RP, Gordon YJ. Emerging fluoroquinolone resistance in bacterial keratitis. Ophthalmology 1999;106:1313-18.

6 Tuft SJ, Mathieson M. In vitro antibiotic resistance in bacterial keratitis in London. Br J Ophthalmol 2000;84:687-91.

7 Hoban DJ, Dawn GV, Fluit AC, et al. Worldwide prevalence of anti-microbial resistance in Streptococcus pneumonae in the sentry anti-microbial surveillance programme 1997-1999. Clin Infect Dis 2001;32/Suppl 2):S81-93

8 Diekema DJ, Pfaller MA, Schmidtz FJ, et al. Survey Infections due to Staphylococcus. Clin Infect Dis 2001;32(Suppl 2):S1 14-32

9 Anon. Moxifloxacin. A journey towards improving anti-microbial therapy. Clin Infect Dis 2001;32(Suppl 1)

10 Gales AC, Jones RN, Turnbridge J, et al.

Characterisation of Pseudomonas aeruginosa isolates: occurrence rates, anti-microbial susceptibility patterns and molecular typing in the Global Sentry Anti-Microbial Surveillance Programme 1997-1999. Clin Infect Dis 2001;32(Suppl 2):S146-55.

11 Davison CR, Tuft SJ, Dart JKG. Conjunctival necrosis after administration of topical fortified aminoglycosides. Am J Ophthalmol 1991;111:690-3.

12 Ofloxacin Study Group. Ofloxacin monotherapy for the primary treatment of microbial keratitis. Ophthalmology 1997; 104:1902-9.

13 Gangopadhyay N, Daniell MD, Weih LA, et al. Fluoroquinolone and fortified antibiotics for treatment of bacterial corneal ulcers. Br J Ophthalmol 2000;84:378-84.

14 Garg P, Scharmer S, Rayo GN. Ciprofloxacin resistant pseudomonas keratitis. Ophthalmology 1999:106:1319-23.

15 Chaudry NA, Flynn HW, Murray TG, et al. Emerging ciprofloxacin resistant pseudomonas aeruginosa. Am J Ophthalmol 1999;128:509-10.

16 O'Brien TP, Maguire MG, Ink NE, et al. Efficacy of ofloxacin versus cephazolin and tobramycin in the therapy for bacterial keratitis. Arch Ophthalmol 1995:113:1257-65.

17 Hyndiuk RA, Eiferman RA, Caldwell DR, et al. Comparison of ciprofloxacin ophthalmic solution $0.3 \%$ to fortified tobramycin cephazolin in treating bacterial corneal ulcers. Ophthalmology 1996;103:1854-63.

18 Wilhelmus KR, Hyndiuk RA, Caldwell DR, et al. $0.3 \%$ Ciprofloxacin ophthalmic ointment in the treatment of bacterial keratitis. Arch Ophthalmol 1993;111:1210 18.

19 Mallari PLT, McCarty DJ, Daniell MD, et al. Increased incidence of corneal perforation after topical fluoroquinolone treatment for microbial keratitis. Am J Ophthalmol 2001;131:131-3.

20 Pollack GA, McCarty DJ, McKelvie PA, et al. The in vivo effects of fluoroquinolones on rabbit corneas. ARVO Abstract 2001 